\title{
Dynamic contrast-enhanced magnetic resonance imaging for monitoring neovascularization during bone regeneration-a randomized in vivo study in rabbits
}

\author{
L. A. R. Righesso ${ }^{1}$ - M. Terekhov ${ }^{2}$ - H. Götz ${ }^{3}$ - M. Ackermann ${ }^{4}$ - T. Emrich T,6,7 $~$ L. M. Schreiber $^{2}$ • W. E. G. Müller ${ }^{8}$. \\ J. Jung ${ }^{9}$ • J. P. Rojas ${ }^{10}$ - B. Al-Nawas ${ }^{1}$
}

Received: 6 December 2020 / Accepted: 12 March 2021 / Published online: 30 March 2021

(C) The Author(s) 2021

\begin{abstract}
Objectives Micro-computed tomography $(\mu-\mathrm{CT})$ and histology, the current gold standard methods for assessing the formation of new bone and blood vessels, are invasive and/or destructive. With that in mind, a more conservative tool, dynamic contrastenhanced magnetic resonance imaging (DCE-MRI), was tested for its accuracy and reproducibility in monitoring neovascularization during bone regeneration. Additionally, the suitability of blood perfusion as a surrogate of the efficacy of osteoplastic materials was evaluated.

Materials and methods Sixteen rabbits were used and equally divided into four groups, according to the time of euthanasia ( 2,3 , 4 , and 6 weeks after surgery). The animals were submitted to two 8-mm craniotomies that were filled with blood or autogenous bone. Neovascularization was assessed in vivo through DCE-MRI, and bone regeneration, ex vivo, through $\mu$-CT and histology. Results The defects could be consistently identified, and their blood perfusion measured through DCE-MRI, there being statistically significant differences within the blood clot group between 3 and 6 weeks $(p=0.029)$, and between the former and autogenous bone at six weeks $(p=0.017)$. Nonetheless, no significant correlations between DCE-MRI findings on neovascularization and $\mu$-CT $(r=-0.101,95 \%$ CI $[-0.445 ; 0.268])$ or histology $(r=0.305,95 \%$ CI $[-0.133 ; 0.644])$ findings on bone regeneration were observed.

Conclusions These results support the hypothesis that DCE-MRI can be used to monitor neovascularization but contradict the premise that it could predict bone regeneration as well.
\end{abstract}

Keywords Animal experimentation - Bone regeneration - Multiparametric magnetic resonance imaging · Neovascularization, physiologic $\cdot$ Tissue engineering $\cdot$ Translational medical research

Clinical relevance A reasonable extrapolation to clinical applications is DCE-MRI could be used not as a substitute, but as a complement to CT findings, providing information on the viability of the osteoid formed.

L. A. R. Righesso

righesso@protonmail.com

1 Clinic for Oral and Maxillofacial Surgery and Plastic Surgery, University Medical Center of the Johannes Gutenberg University Mainz, Augustusplatz 2, 55131 Mainz, Germany

2 Molecular and Cellular Imaging, Comprehensive Heart Failure Center, University Hospital Würzburg, Josef-Schneider-Strasse 2, 97080 Würzburg, Germany

3 Cell Biology Unit, University Medical Center of the Johannes Gutenberg University Mainz, Langenbeckstrasse 1, 55131 Mainz, Germany

4 Institute of Functional and Clinical Anatomy, University Medical Center of the Johannes Gutenberg University Mainz, Johann-Joachim-Becher-Weg 13, 55128 Mainz, Germany
5 Department of Radiology, University Medical Center of the Johannes Gutenberg University Mainz, Langenbeckstrasse 1, 55131 Mainz, Germany

6 Medical University of South Carolina, 171 Ashley Avenue, Charleston, SC 29425, USA

7 German Center for Cardiovascular Research (DZHK), Partner-Site Rhine-Main, Potsdamer Strasse 58, 10785 Berlin, Germany

8 Institute of Physiological Chemistry, University Medical Center of the Johannes Gutenberg University Mainz, Duesbergweg 6, 55128 Mainz, Germany

9 Department of Oral and Maxillofacial Surgery, School of Dentistry, Kyung Hee University, 23, Kyung Hee Dae-ro, Dongdaemun-gu, Seoul 02447, Republic of Korea

10 Private Practice, Av. La Dehesa, 181 Santiago, Chile 


\section{Introduction}

Tissue engineering is based on three pillars: reparative cells that can form a functional matrix; an appropriate scaffold for transplantation and support; and bioreactive molecules, like cytokines and growth factors, which can support and coordinate the formation of the desired tissue [1]. However, more recent studies show that, without the establishment of a vascular network, incorporation of engineered constructs simply does not take place [2]. Hence, research on new ways of improving construct vascularity is gaining momentum [3-5].

Many of the studies on the topic, however, rely on invasive and/or destructive tools, such as histology and radiationemitting imaging, to assess bone regeneration and neovascularization $[6,7]$. Though accurate, these methods present a few downsides. Histology, for one, requires a surgical specimen. In clinical studies, when it is possible to obtain one, additional harm is usually caused to patients, rendering repeated measurements virtually impossible. As for animal studies, subjects must be euthanized, precluding an intra-individual longitudinal assessment. Alternatively, an inter-individual analysis could be performed, but that would imply sacrificing animals at each time point, increasing sample size and, consequently, costs, but more importantly, clashing with the 3Rs of animal research (replacement, reduction, and refinement). Moreover, for histological analysis, the surgical specimen must be sliced, rendering further examinations by other methods difficult or impossible. Lastly, results are reported by sections and, for volumetric determinations, the results are often estimated by interpolation [8].

More recently, micro-computed tomography $(\mu-\mathrm{CT})$ has been described for monitoring neovascularization and bone regeneration [9]. Among its advantages, it can be cited that it not only allows assessing both outcomes but also allows doing so three-dimensionally. Moreover, it is a nondestructive method [10]. Nevertheless, the ionizing radiation emitted during $\mu$-CT imaging becomes hazardous when research subjects need to be submitted to repeated exams [11]. Also, in many instances, similarly to histologic analysis, to perform a $\mu-\mathrm{CT}$, a surgical specimen must be obtained and the animal euthanized, which also precludes an intraindividual longitudinal assessment.

As a non-invasive imaging technique, magnetic resonance imaging (MRI) is suitable for in vivo longitudinal evaluation of tissue repair following grafting procedures. Non-invasive dynamic MRI following the administration of a contrast agent can provide functional information on the microcirculation. This technique, also known as dynamic contrast-enhanced MRI (DCE-MRI), has shown potential in a wide range of clinical applications, especially in oncology, where physiological properties, such as blood flow, blood volume, and vessel permeability need to be determined quantitatively [12] However, despite its well-established role in assessing the microvasculature in pre- and clinical and studies, the use of DCE-MRI in tissue engineering has been little explored.

The aim of the present study was to assess the accuracy and reproducibility of DCE-MRI in monitoring neovascularization during bone regeneration and to evaluate whether the initial area under the contrast concentration versus time curve at $140 \mathrm{~s}\left(\mathrm{IAUC}_{140}\right)$, a DCE-MRI pharmacokinetic parameter of blood perfusion, is a reliable surrogate of the efficacy of osteoplastic materials.

\section{Materials and methods}

This research was developed in an exploratory experimental design, with randomized sampling, a control group, and blinded evaluators.

The experiment complied with the fundamental principles of good laboratory practice, having received ethical approval from the Tierschutzkommission Rheinland-Pfalz (G 15-1084).

\section{Population and sample}

For this study, 16 skeletally mature (5-6 months old) New Zealand white female rabbits, weighing $3.5-4.5 \mathrm{~kg}$, were used. Animals were randomly divided into four groups of four, according to the time of euthanasia $(2,3,4$, and 6 weeks after surgery), using an online free calculator (www.graphpad. com/quickcalcs/randomize1/, GraphPad Software, San Diego, USA).

\section{Facilities}

Throughout the experiment, animals were housed under satisfactory conditions, in closed, ventilated shelves, with filtered airflow, temperature, and humidity control, under cold light and supervision of veterinary staff. They were fed solid ration, appropriate to them, and water ad libitum. They were kept in standardized cages, labeled, and identified with the group number to which they belonged, date of birth, weight, date of surgery, date of death, names of researcher, and advisor. Cages were cleaned three times a week, with running water, soap, and disinfectant.

\section{Surgical procedure}

Surgery was performed under rigorous aseptic conditions and general anesthesia. The latter was obtained with an intramuscular (IM) injection of a combination of medetomidine $(0.2$ $\mathrm{mg} / \mathrm{kg})$, midazolam $(1 \mathrm{mg} / \mathrm{kg})$, and fentanyl $(0.02 \mathrm{mg} / \mathrm{kg})$.

Once the animal was unconscious, the surgical site was shaved, washed with $2 \%$ iodine antiseptic solution and then, infiltrated with $1.8 \mathrm{ml}$ of $2 \%$ lidocaine HCL with 1:100.000 


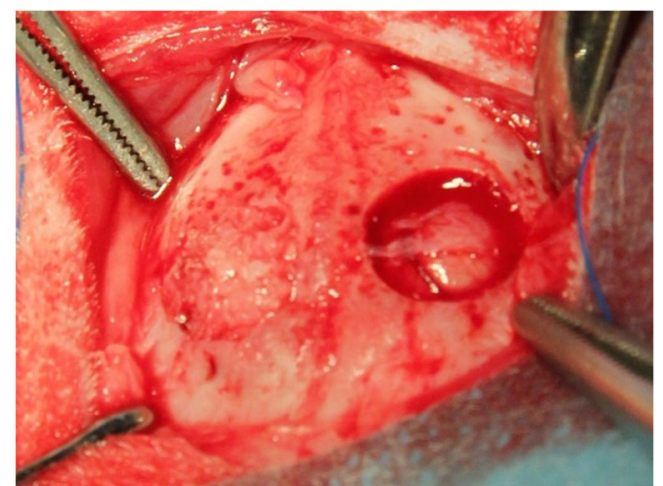

Fig. 1 Craniotomies prepared in the parietal bones. On the left side, the defect was grafted with autogenous bone, while on the right side, it was left to fill with blood

epinephrine. A coronal incision was made, and the soft tissues reflected to expose the calvarium. Using a surgical handpiece with a diamond trephine, while irrigating with copious physiological saline, two craniotomies ( $8 \mathrm{~mm}$ diameter) were prepared in the parietal bones. Care was taken not to tear the dura mater.

One defect was grafted, until visually filled, with autogenous bone chips, obtained through the morselization of the trephined bone with a bone scraper (Geistlich SafeScraper TWIST, Geistlich Pharma AG, Wolhusen, Switzerland), while the other defect was left to fill with blood and served as control (Fig. 1).

Treatments were allocated to the craniotomies, according to a $4 \times 2$ Latin squares design. Next, a resorbable, collagen membrane (Geistlich Bio-Gide ${ }^{\circledR}$, Geistlich Pharma AG, Wolhusen, Switzerland) was used to protect the initial coagulum and prevent particle migration or soft tissue ingrowth into the grafted sites. Finally, soft tissues were approximated and sutured in layers.

Immediately after surgery, the animals were administered subcutaneous injections of the antibiotic enrofloxacin $2.5 \%$ $(0.4 \mathrm{ml} / \mathrm{kg})$, the anti-inflammatory carprofen $50 \mathrm{mg} / \mathrm{ml}(0.1$ $\mathrm{ml} / \mathrm{kg})$, and the opioid analgesic buprenorphine $(0.07 \mathrm{ml} / \mathrm{kg})$. This was repeated once a day, for the next 3 days.

\section{Dynamic contrast-enhanced magnetic resonance imaging}

Image acquisition was performed under general anesthesia, using the same protocol as for surgery. Imaging sessions were held at 2, 3, 4, and 6 weeks after surgery, one group at a time, according to the established time of euthanasia. The device used was the 3-Tesla MAGNETOM® Prisma scanner (Siemens Healthineers GmbH, Erlangen, Germany), with a 4-channel flexible receive array and a body transmit coil. Animals were placed in prone position and an axial orientation was used for all the slices. Overview protondensity contrast images in the sagittal plane were acquired to plan slices for the subsequent DCE-MRI. The pulse sequence used was turbo spin-echo, with TR/TE $=1100 / 39$ $\mathrm{ms}$, echo train length $=29$, slice thickness $=0.5 \mathrm{~mm}$, number of slices $=96$, image matrix $=448 \times 275$, and field-ofview $=219 \times 150 \mathrm{~mm}$. Additionally, high-resolution anatomical images in the transversal plane were acquired at the operated area using a turbo spin-echo sequence, with $\mathrm{TR} / \mathrm{TE}=600 / 15 \mathrm{~ms}$, field-of-view $=89 \times 89 \mathrm{~mm}$, matrix $438 \times 336$, voxel size $=0.2 \times 0.25 \times 1.5 \mathrm{~mm}$, and 16 slices covering the operated area (Fig. 2). (For more details, please refer to Table 1).

Anatomy protocols (turbo spin-echo columns 1 and 2) used for localization of the slab. Column \#3 provides parameters for perfusion data acquisition

Dynamic T1-weighted images were acquired using a fast SGRE pulse sequence, with TR $/ \mathrm{TE}=15 / 1.8 \mathrm{~ms}$, Flip Angle $=$ 750 , image matrix $=128 \times 128$, field-of-view $=62 \times 62 \mathrm{~mm}$, voxel size $=0.48 \times 0.48 \times 1 \mathrm{~mm}$, number of slices $=10$, and a parallel imaging acceleration factor $R=2$. After the acquisition of five baseline images, gadolinium diethylenetriamine pentaacetic acid (Gd-DTPA) was administered as a rapid bolus $(0.1 \mathrm{mmol}$ of $\mathrm{Gd} / \mathrm{kg})$ through the ear vein and imaging continued for 6 min post-injection with an effective temporal resolution of $8.2 \mathrm{~s}$.

After the imaging session, still under general anesthesia, the animals were sacrificed through an anesthetic overdose.
Table 1 Parameters of measurement protocols

\begin{tabular}{llll}
\hline Parameter & Anatomy (TSE) & Anatomy (TSE) & Perfusion (GRE 3D) \\
\hline Orientation & Transversal & Sagittal & Transversal \\
Repetition time (ms) & 600 & 1100 & 15 \\
Echo train length & 4 & 29 & N/A \\
Echo time (ms) & 15 & 39 & 1.79 \\
Matrix size & $356 \times 448$ & $356 \times 448$ & $128 \times 128$ \\
Slice thickness (mm) & 1.5 & 0.5 & 1 \\
Field-of-view (mm) & $89 \times 89$ & $150 \times 219$ & $62 \times 62$ \\
Number of slices & 16 & 96 & 10 \\
Measurements & 1 & 1 & 20
\end{tabular}


Fig. 2 a Sagittal slice of highresolution anatomical image delineating one of the surgical sites. The yellow rectangle represents the transversal slab used for planning the DCE-MRI slices. $\mathbf{b}$ Coronal slice of a high-resolution anatomical image used to confirm localization. This slice corresponds to the dashed line at the center of the rectangle in a. The yellow arrow identifies a specific point that is shown in two different planes
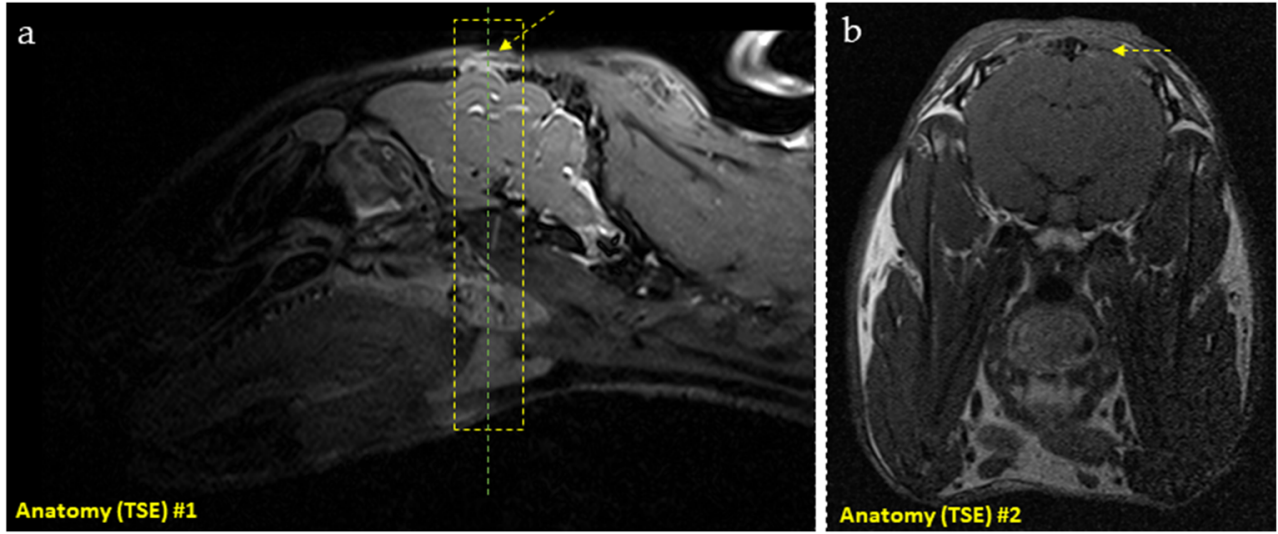

The calvarium was excised and placed in $10 \%$ formalin solution for subsequent $\mu-\mathrm{CT}$ and histological procedures.

Perfusion analysis was performed by an experienced radiologist (T.E.), using a dedicated software solution CVI42@ (Circle Cardiovascular Imaging Inc., Calgary, Canada). Initially, high-resolution T2 images were used to locate the defects and their borders. Then, these anatomical images were matched to the perfusion datasets in the same software to allow optimal contouring of the region of interest (ROI). Contours were drawn manually for both defects, as well as for the reference tissues, the brain at brain stem level, and the right masseter muscle. Manual contouring was performed for every slice and time point in the perfusion dataset. Every defect consisted of ten slices, which included both defects in coronal angulation. In questionable cases, a consensus ROI was drawn after interdisciplinary discussion (T.E. and L.A.R.R.). Motion and artifacts were carefully excluded. Furthermore, potential confounders of perfusion, like vessels, were carefully eliminated. Perfusion curves and measurement included the relative signal intensity over time.

Fig. 3 Flow chart of the study design
The initial area under the curve at $140 \mathrm{~s}\left(\mathrm{IAUC}_{140}\right)$ was then calculated for each ROI (left defect, right defect, brain tissue, muscle tissue) on each slice using IBM SPSS Statistics for Windows, version 23.0 (IBM Corp., Armonk, NY, USA). Finally, a mean value was established for each ROI.

\section{Micro-computed tomography}

Imaging was performed using the high-resolution micro-computed tomography scanner $\mu-C T 40$ (Scanco Medical AG, Brüttisellen, Switzerland). Samples containing the whole calvarium were scanned under the following specifications: the specimen tube of the $\mu$-CT scanner was run with the X-ray tube operating at $70 \mathrm{kVp}$ and $114 \mu \mathrm{A}$, with a focal spot size of $6 \mu \mathrm{m}$. Per rotation, 1000 pictures were taken with an integration time of $300 \mathrm{~ms}$ and $2048 \times 2048$ pixels. Reconstruction resulted in 208 pictures/probe with a voxel size of $18 \mu \mathrm{m}$, corresponding to a layer thickness of $3 \mu \mathrm{m}$ and height of 6 $\mathrm{mm}$. Thereby, the whole calvaria could be reconstructed. Bone volume fraction (BVF) was then computed by dividing

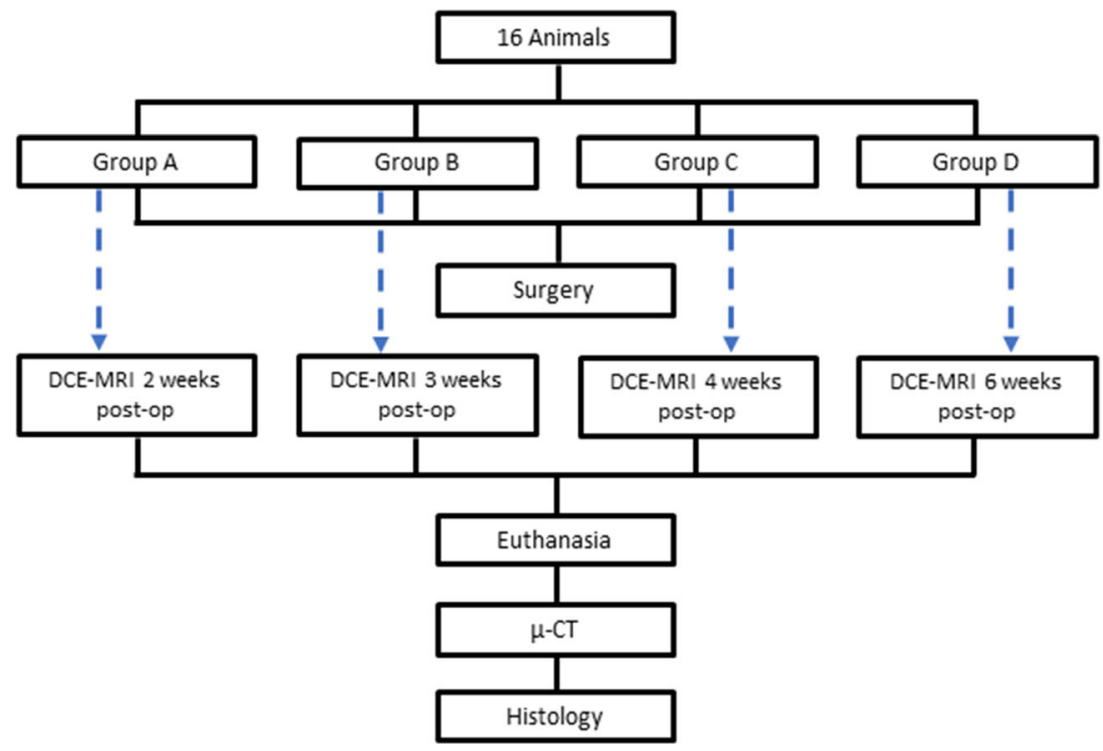


Fig. 4 A coronal slice from one animal from each group was selected for visual comparison among different time points. The 5 th slice from anterior to posterior was picked for each animal, corresponding to the center of the defect. On the right, the perfusion curves can be seen. In red, values for the right defect; in green, for the left one; in orange, for an ROI of the brain; and, in yellow, for the right masseter muscle
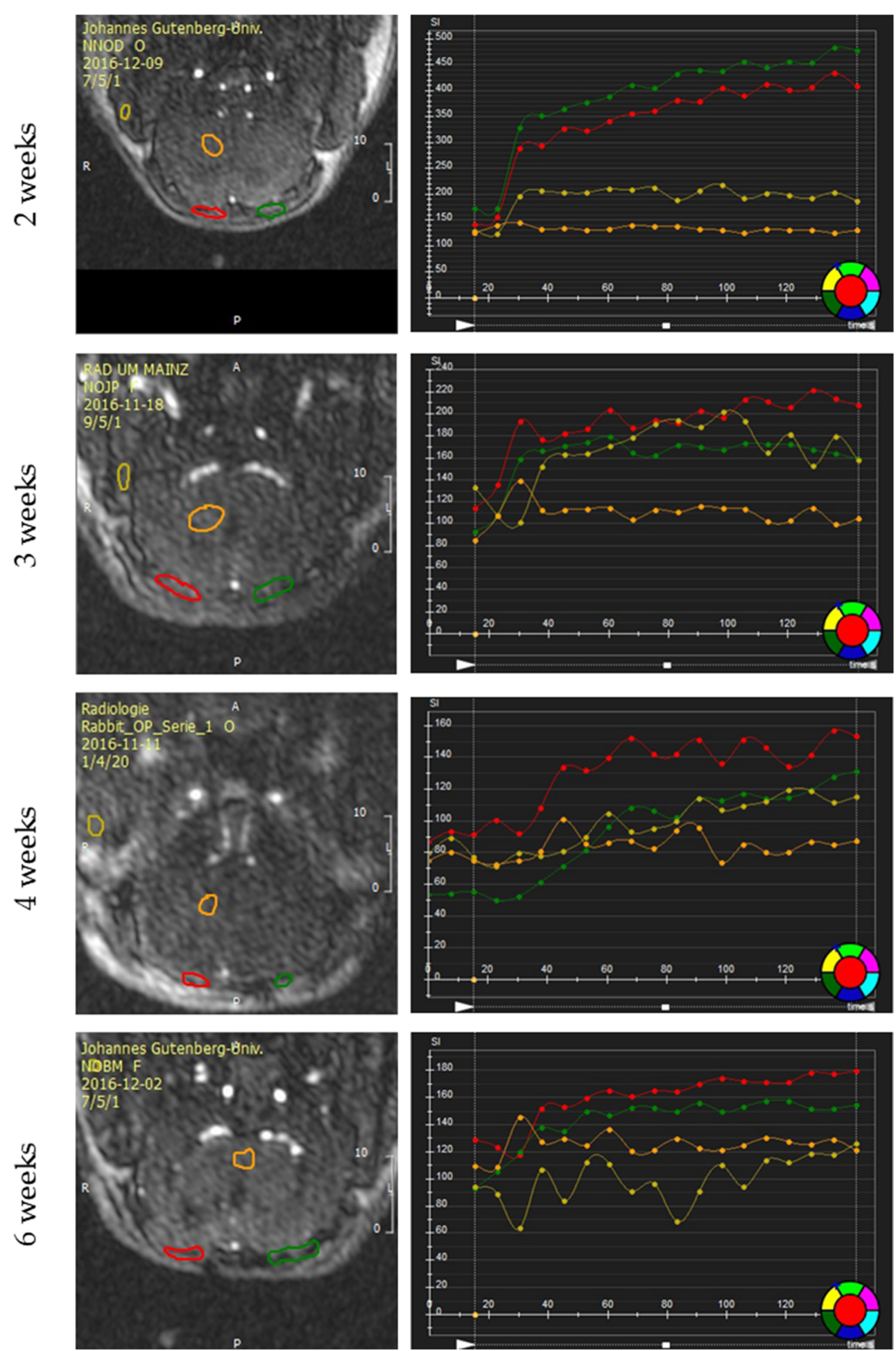

the bone volume by a specified cylindrical total volume, defined by the initial drilling diameter $(8 \mathrm{~mm})$ and the thickness of the calvaria. This cylinder was orthogonally projected onto the bone defect and the calculation was performed using the Scanco Medical Software (Scanco Medical AG, Brüttisellen, Switzerland).

\section{Histological analysis}

After $\mu-\mathrm{CT}$, the samples were prepared for histological analysis. Mineralized samples were directly embedded in Technovit@ 7200 VLC (Kulzer GmbH, Hanau, Germany). Sectioning and grinding of Technovit ${ }$ embedded samples 
Table 2 Comparison of IAUC $_{140}$ between the two treatments at different time points

\begin{tabular}{llll}
\hline Time (weeks) & Estimate & Standard error & $p$ value \\
\hline 2 & -331 & 1393 & 0.811 \\
3 & -1862 & 2572 & 0.469 \\
4 & -6257 & 2921 & 0.032 \\
6 & -8107 & 3422 & $0.017^{\mathrm{a}}$ \\
\hline
\end{tabular}

Statistically significant differences in IAUC140 between the two treatments at different time points are represented by letters. $\mathrm{a}=0.017$

were performed as described by Donath [13]. The slices were then stained with toluidine blue and analyzed under conventional qualitative bright-field microscopy analysis. For quantification of new bone formation (NBF), the quantity of newly formed bone was calculated as the percentage fraction of the new bone area to the total defect area. A histomorphometric analysis of the tissue area on the pictures was performed using ImageJ (NIH). Prior to that, the pictures were anonymized using the extension AbleBits.com random generator, for Microsoft ${ }^{\circledR}$ Excel ${ }^{\circledR} 2013$ (Microsoft Corporation, Redmond, USA), so the evaluator could assess them blindly.

\section{Statistical analysis}

Data were analyzed using IBM SPSS Statistics for Windows, version 23.0 (IBM Corp., Armonk, NY, USA). The bone defect was set as the statistical unit. A generalized estimating equation test was used to compare treatment and time of euthanasia using exchangeable correlation structure, accounting for correlations between repeated measurements on the same animal. Interactions were included in the model, if necessary, and mean

Table 3 Comparison of IAUC $_{140}$ between time points, according to treatment

\begin{tabular}{lllll}
\hline Time (weeks) & Treatment & Estimate & Standard error & $p$ value \\
\hline $2-3$ & Autogenous bone & 13,540 & 7310 & 0.383 \\
$2-4$ & Autogenous bone & 14,136 & 8034 & 0.448 \\
$2-6$ & Autogenous bone & 11,978 & 7577 & 0.683 \\
$3-4$ & Autogenous bone & 776 & 4749 & 1.000 \\
$3-6$ & Autogenous bone & -1562 & 3926 & 1.000 \\
$4-6$ & Autogenous bone & -2337 & 5150 & 1.000 \\
$2-3$ & Blood clot & 12,009 & 7929 & 0.779 \\
$2-4$ & Blood clot & 8390 & 8301 & 1.000 \\
$2-6$ & Blood clot & 4202 & 8301 & 1.000 \\
$3-4$ & Blood clot & -3619 & 2775 & 1.000 \\
$3-6$ & Blood clot & -7087 & 2774 & 0.029 \\
$4-6$ & Blood clot & -4188 & 3705 & 1.000 \\
\hline
\end{tabular}

Statistically significant differences in IAUC140 between time points, according to treatment, are represented by letters. $b=0.029$ values were compared using simple effects analyses with a post hoc Bonferroni adjustment. Correlations between $\mu-\mathrm{CT}$ and DCE-MRI, histology, and DCE-MRI, $\mu$-CT, and histology were assessed using Spearman's rank correlation coefficient.

In Fig. 3, the study design is summarized in graphical form to facilitate comprehension, as recommended in the ARRIVE guidelines [14].

\section{Results}

\section{Dynamic contrast-enhanced magnetic resonance imaging}

\section{Descriptive analysis}

The defects were identifiable even prior to contrast agent injection, appearing in coronal slices as concave disk-shaped structures, being hypointense in $\mathrm{T} 1 \mathrm{w}$ images and hyperintense in $\mathrm{T} 2 \mathrm{w}$ ones. After contrast agent injection, the blood clot group showed a slightly more pronounced signal intensity, especially from week 3. Also, a hyperintense signal moving in a centripetal fashion was observed in both groups. In the first weeks, uptake of contrast medium was fast and washout, slow, and its distribution was concentrated in the periphery of the defect. With time, the development of a vascular network led to a more sustainable uptake and washout and to a more even distribution of the contrast agent inside the defect. A comparison between animals from each group is presented in Fig. 4.

\section{IAUC $_{140}$}

Statistically significant differences were found, when comparing treatments, at 6 weeks, when blood clot showed a statistically higher mean $\mathrm{IAUC}_{140}$ than autogenous bone $(p=0.017)$ (Table 2 and Fig. 5), and, when comparing time points, in the blood clot group, between weeks 3 and $6(p=0.029)$ (Table 3 and Fig. 5).

\section{Micro-computed tomography}

\section{Bone volume fraction}

Statistically significant differences were found, when comparing treatments, at weeks 3,4 , and 6 , when autogenous bone showed a statistically higher mean BVF than blood clot $(p<$ $0.001, p<0.001, p=0.003$, respectively) (Table 4 and Fig. 6), and, when comparing time points, in the autogenous bone group, between weeks 3 and $4(p=0.039)$ and 4 and $6(p=$ $0.005)$. As for the blood clot group, statistically significant differences were found between weeks 2 and $3(p<0.001)$ and 3 and $4(p=0.003)$ (Table 5 and Fig. 6). 
Fig. 5 Effect of treatment on IAUC $_{140}$ over time. Dots represent the mean value, while whiskers represent the standard error. Letters represent statistically significant differences, as seen in Tables 2 and 3

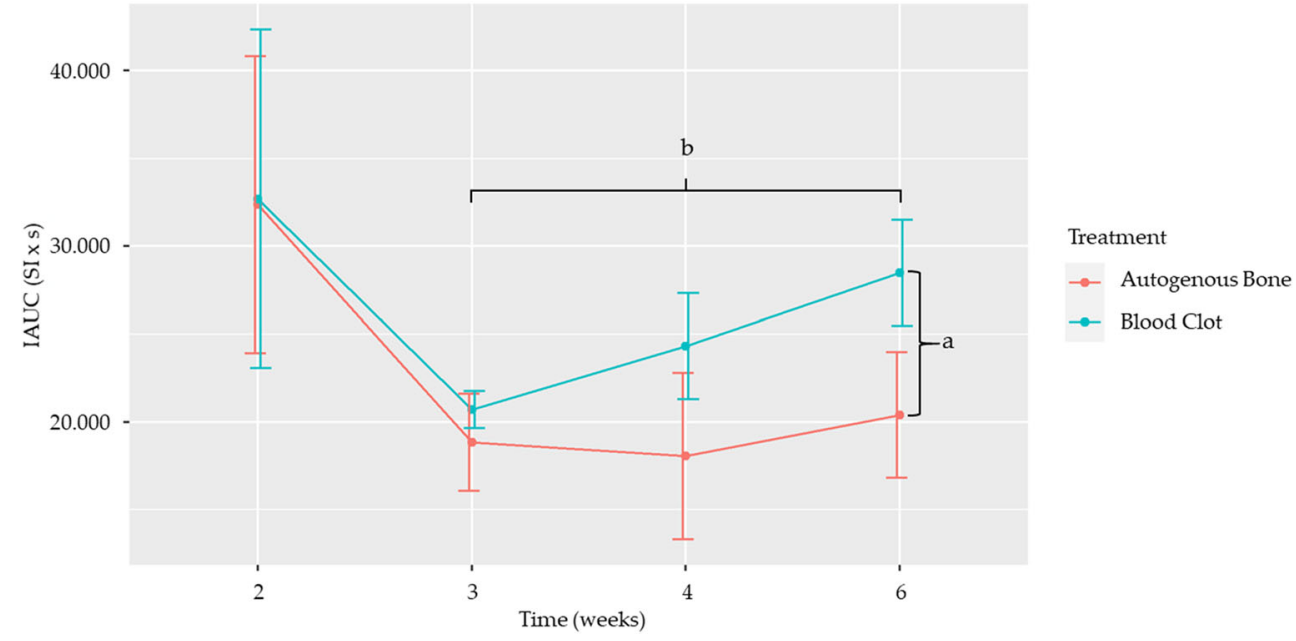

\section{Histological analysis}

\section{New bone formation}

Statistically significant differences were found, when comparing treatments, at 6 weeks, when autogenous bone showed a statistically higher mean NBF than blood clot $(p=0.023)$ (Table 6 and Fig. 7); and, when comparing time points, in the blood clot group, between weeks 2 and $3(p=<0.001)$ and 2 and $6(p=<0.001)$ (Table 7 and Fig. 7).

\section{Correlations}

There was no statistically significant correlation between $\mu$-CT and DCE-MRI ( $r=-0.101,95 \%$ CI $[-0.445 ; 0.268])$, and histology and DCE-MRI $(r=0.305,95 \%$ CI $[-0.133 ; 0.644])$, nor between $\mu$-CT and histology $(r=0.237,95 \%$ CI $[-0.204$; 0.599]) (Figs. 8, 9, and 10).

In Fig. 11, a montage is presented for visual comparison among the three assessment tools.

Table 4 Comparison of BVF between the two treatments at different time points

\begin{tabular}{llll}
\hline Time (weeks) & Estimate & Standard error & $p$ value \\
\hline 2 & 0.127 & 0.032 & $<0.001^{\mathrm{c}}$ \\
3 & 0.126 & 0.027 & $<0.001^{\mathrm{d}}$ \\
4 & 0.063 & 0.035 & 0.069 \\
6 & 0.154 & 0.052 & $0.003^{\mathrm{e}}$ \\
\hline
\end{tabular}

Statistically significant differences in BVF between the two treatments at different time points are represented by letters. $\mathrm{c}, \mathrm{d}<0.001$

\section{Discussion}

In this viability study, neovascularization could be successfully monitored during bone regeneration using DCE-MRI. We were able to consistently identify the two craniotomies and measure the blood perfusion within them.

The blood clot group showed a higher mean IAUC $_{140}$ throughout the experiment, even though a statistically significant difference between treatments was only observed at 6 weeks. One possible explanation for this phenomenon could be the higher amount of empty space in the defects left to fill with blood, leaving more room for blood vessels to form. Also, this group lacks the osteoconductive, osteoinductive, and osteogenic capacities of autogenous bone, depending entirely on the

Table 5 Comparison of BVF between time points, according to treatment

\begin{tabular}{lllll}
\hline Time (weeks) & Treatment & Estimate & Standard error & $p$ value \\
\hline $2-3$ & Autogenous bone & -0.046 & 0.041 & 1.000 \\
$2-4$ & Autogenous bone & 0.064 & 0.043 & 0.806 \\
$2-6$ & Autogenous bone & -0.095 & 0.048 & 0.298 \\
$3-4$ & Autogenous bone & 0.111 & 0.041 & $0.039^{\mathrm{f}}$ \\
$3-6$ & Autogenous bone & -0.048 & 0.046 & 1.000 \\
$4-6$ & Autogenous bone & -0.159 & 0.048 & $0.005^{\mathrm{g}}$ \\
$2-3$ & Blood clot & -0.047 & 0.011 & $<0.001^{\mathrm{h}}$ \\
$2-4$ & Blood clot & 0.001 & 0.012 & 1.000 \\
$2-6$ & Blood clot & -0.068 & 0.034 & 0.288 \\
$3-4$ & Blood clot & 0.049 & 0.014 & $0.003^{\mathrm{i}}$ \\
$3-6$ & Blood clot & -0.020 & 0.035 & 1.000 \\
$4-6$ & Blood clot & -0.069 & 0.035 & 0.298 \\
\hline
\end{tabular}

Statistically significant differences in BVF between time points, according to treatment, are represented by letters. $g=0.005, \mathrm{~h}<0.001, \mathrm{i}=0.003$ 
Fig. 6 Effect of treatment on bone volume fraction over time. Dots represent the mean value, while whiskers represent the standard error. Letters represent statistically significant differences, as seen in Tables 4 and 5



surrounding host bone and periosteum for repair, which may trigger a longer and more pronounced inflammatory response.

Regarding the time of euthanasia, a statistically significant difference was found within the blood clot group between 3 and 6 weeks, while none was seen for autogenous bone. On a closer inspection, a trend can be observed. Between the second and third weeks, a sharp decrease in IAUC $_{140}$ was seen in both treatment groups, consistent with a transition from the inflammatory to the proliferative phase of wound healing. After that, a steady growth was observed in the blood clot group until week 6 , while in the autogenous bone group there was a slight decrease between weeks 3 and 4, after which point a growth was also observed, albeit not as pronounced as in the blood clot group. These findings contrast with Beaumont et al. [15], who observed a fast drop in perfusion from week 1 to 2 and, then, a stabilization until week 6 , after which point perfusion dropped steadily until week 12, the latter being attributed to bone remodeling. Our follow-up was not as long, but a similar tendency from week 6 could be anticipated, since, as the authors point out, that is consistent with established models of bone regeneration [16]. Despite sharing the same animal model and one assessment method, the DCE-MRI, the studies differ on the grafting material and how the IAUC was calculated, which could account for these differences.

Table 6 Comparison of NBF between the two treatments at different time points

\begin{tabular}{llll}
\hline Time (weeks) & Estimate & Standard error & $p$ value \\
\hline 2 & -2.03 & 3.36 & 0.545 \\
3 & 0.157 & 8.47 & 0.985 \\
4 & -2.38 & 7.04 & 0.735 \\
6 & 18.4 & 8.12 & $0.023^{j}$ \\
\hline
\end{tabular}

Statistically significant differences in NBF between the two treatments at different time points are represented by letters. $\mathrm{j}=0.023$
As for correlations between findings of the three assessment tools, no significant correlation was observed between neovascularization and bone regeneration, contradicting the premise that IAUC $_{140}$ could be a biomarker of the efficacy of osteoplastic materials. Woloszyk et al. [17], however, advocate otherwise. In a study using multimodal MRI and $\mu-\mathrm{CT}$ to quantify angiogenesis in eggs implanted with two different types of bone substitutes, the authors found that perfusion capacity (MRI) and total vessel volume $(\mu-\mathrm{CT})$ strongly correlated for both biomaterials, thereby their approach would be suitable to evaluate vascularization patterns and the efficacy of biomaterials. However, bone formation was not evaluated in the study, and thus, it is not possible to infer the efficacy of bone substitutes.

Coming back to our correlations, a positive correlation between DCE-MRI and histomorphometry was observed, albeit not statistically significant, which could be due to the small size

Table 7 Comparison of NBF between time points, according to treatment

\begin{tabular}{lllll}
\hline Time (weeks) & Treatment & Estimate & Standard error & $p$ value \\
\hline $2-3$ & Autogenous bone & 19.28 & 10.52 & 0.401 \\
$2-4$ & Autogenous bone & 17.09 & 7.82 & 0.1729 \\
$2-6$ & Autogenous bone & 6.74 & 10.75 & 1.000 \\
$3-4$ & Autogenous bone & -2.19 & 7.74 & 1.000 \\
$3-6$ & Autogenous bone & -12.54 & 10.69 & 1.000 \\
$4-6$ & Autogenous bone & -10.35 & 8.04 & 1.000 \\
$2-3$ & Blood clot & 21.47 & 4.26 & $<0.001^{\mathrm{k}}$ \\
$2-4$ & Blood clot & 16.74 & 6.98 & 0.098 \\
$2-6$ & Blood clot & 27.14 & 5.19 & $<0.001^{1}$ \\
$3-4$ & Blood clot & -4.73 & 5.73 & 1.000 \\
$3-6$ & Blood clot & 5.68 & 3.33 & 0.530 \\
$4-6$ & Blood clot & 10.40 & 6.45 & 0.640 \\
\hline
\end{tabular}

Statistically significant differences in NBF between time points, according to treatment, are represented by letters. $\mathrm{k}, 1<0.001$ 
Fig. 7 Effect of treatment on new bone formation over time. Dots represent the mean value, while whiskers represent the standard error. Letters represent statistically significant differences, as seen in Tables 6 and 7

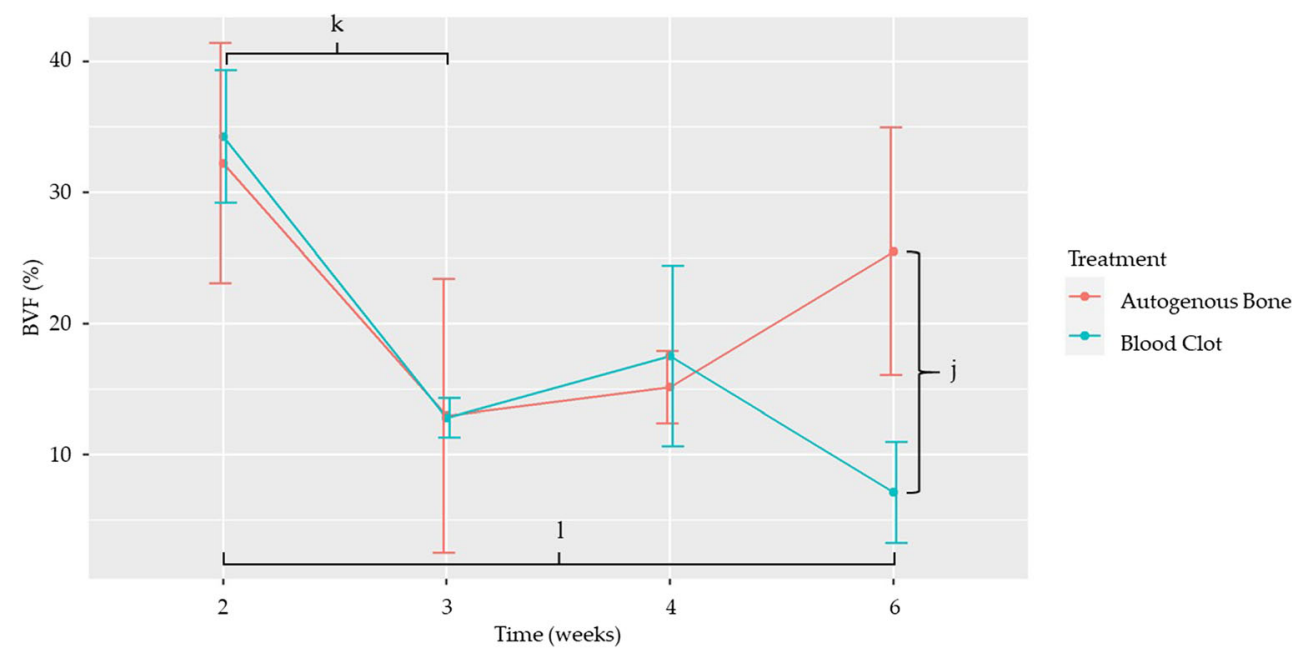

of our sample. That is in line with Sauerbier et al. [18], who observed a correlation between histomorphometry findings on bone regeneration and DCE-MRI findings on neovascularization in a patient submitted to bilateral sinus lifting.

Moving on to an analysis of strong and week points, one strength of the present study is that it used the gold standard material for grafting, autogenous bone, unlike similar studies that used DCE-MRI to monitor neovascularization during bone regeneration $[15,17,18]$. These studies evaluated not only an experimental imaging method but also an experimental grafting material, which adds to the uncertainty and complicates the interpretation of findings. However, using autogenous bone turned out to be a double-edged sword, since both in the histomorphometric and $\mu$-CT analyses, it was difficult to differentiate between grafted bones and newly formed ones. Another strong point of our study was having a negative control group, so that the natural history of bone repair could be established in DCE-MRI, creating a base for comparison for the testing of grafting materials.

Speculating on how our findings could impact clinical practice, it is reasonable to recommend DCE-MRI not as a substitute, but as a complement of $\mathrm{CT}$, as a means to evaluate bone viability, especially in cases of questionable bone quality, like prior to implant placement after bone augmentation or when a pseudoarthrosis is suspected after fracture reduction.

To plan the placement of a dental implant, a panoramic radiography or a cone beam computed tomography is needed to assess bone availability. However, these examinations provide
Fig. 8 Correlation between $\mu$-CT and DCE-MRI findings

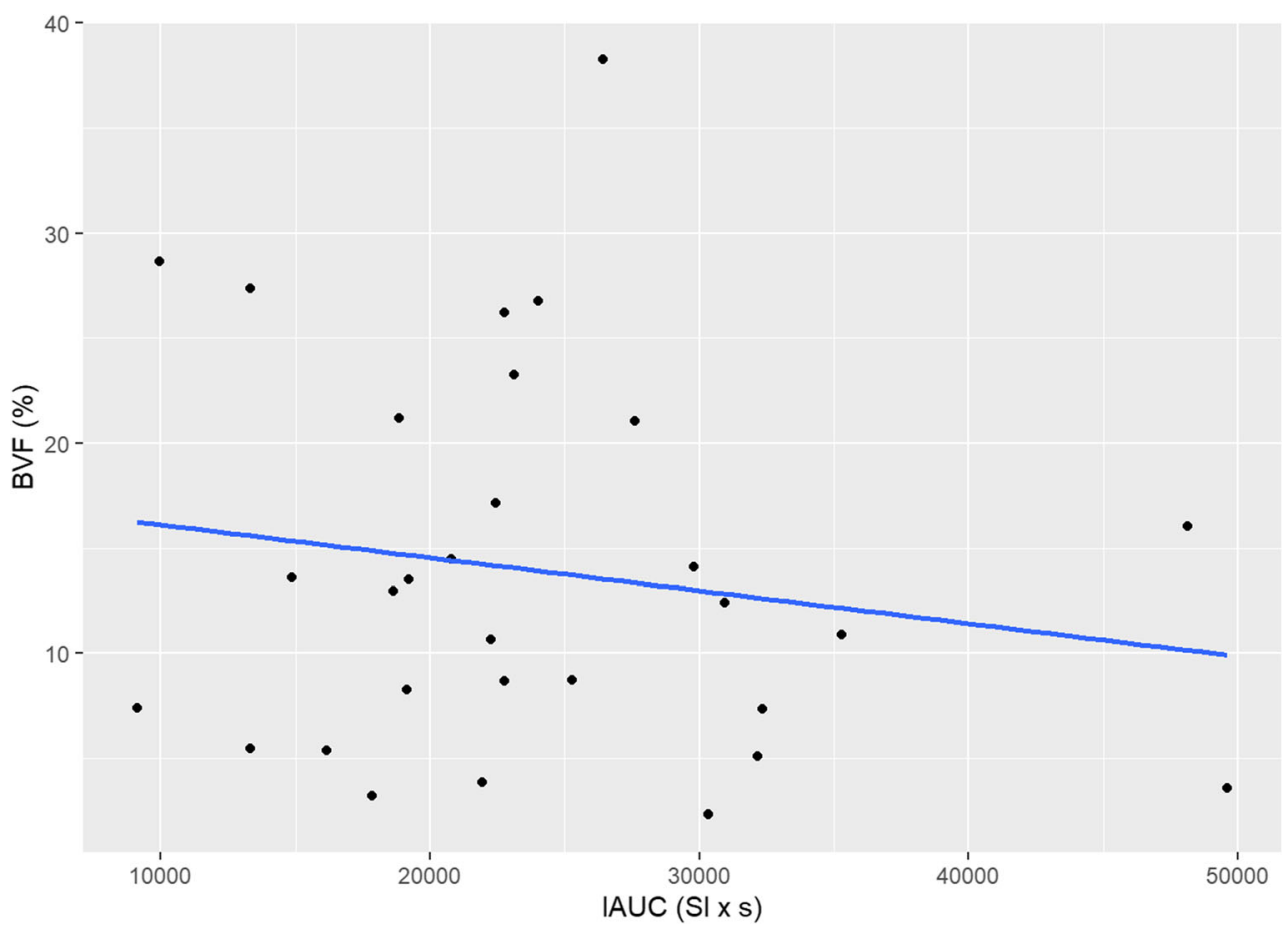


Fig. 9 Correlation between Histology and DCE-MRI findings

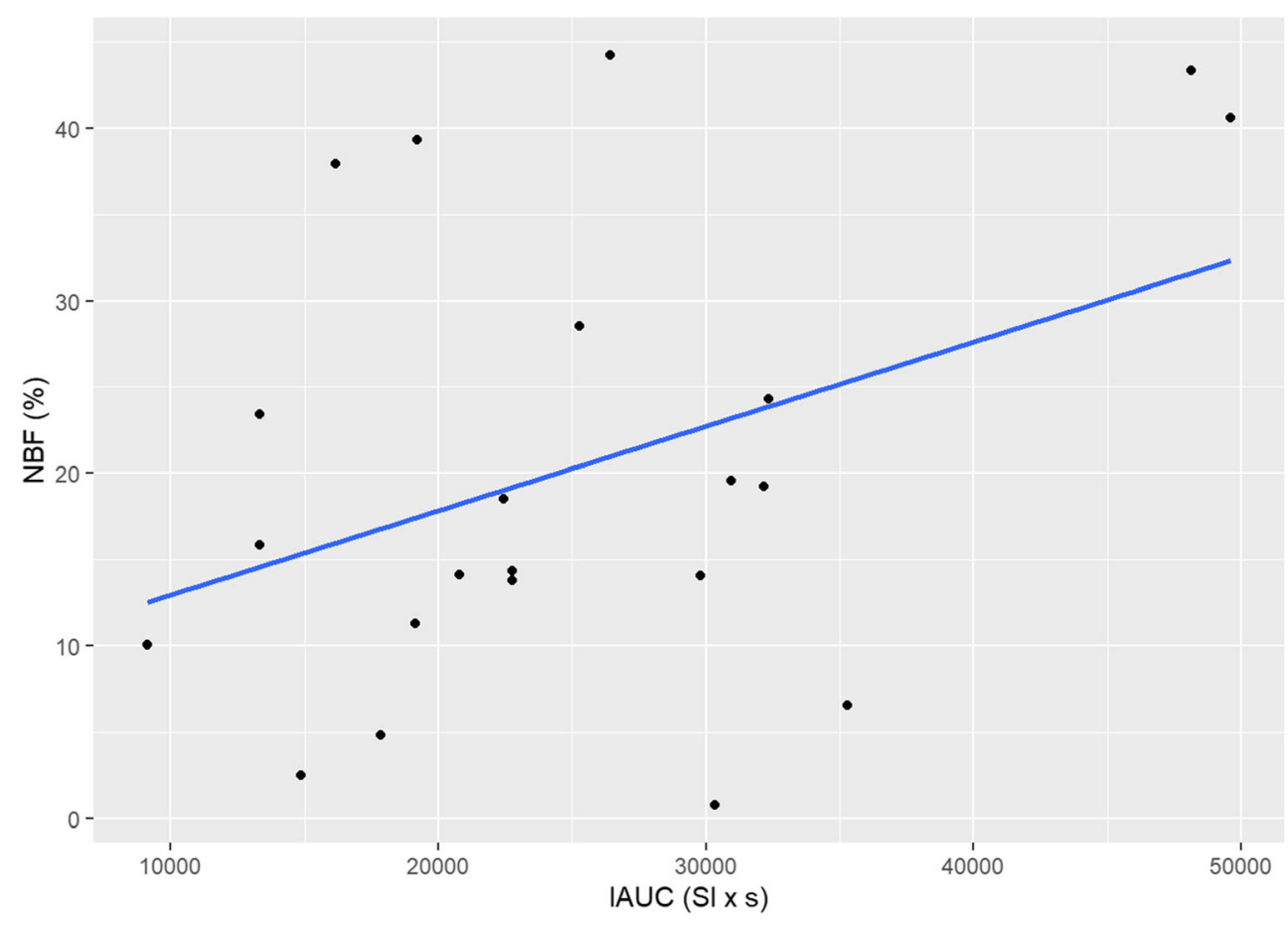

little information on bone quality. In areas where bone augmentation has been performed, it can be hard to differentiate between grafting material and newly formed bone, which can lead to an erroneous assessment of bone viability. Ideally, prior to implant placement, a histological analysis of the bone should be performed in such cases, but this is often unfeasible, since it would result in another surgical procedure. Thus, implants occasionally end up being placed in areas of questionable bone quality, putting osseointegration at risk. What our results suggest is that, in such cases, a DCE-MRI could be performed additionally to the CBCT to evaluate neovascularization where implant placement is planned, providing information on the viability of bone, allowing clinicians to make a better-informed decision, thereby making the treatment more predictable. Sauerbier et al. [18] show that this is feasible and, even though only one patient was evaluated, their results support our recommendation.

Fig. 10 Correlation between $\mu$ $\mathrm{CT}$ and Histology findings

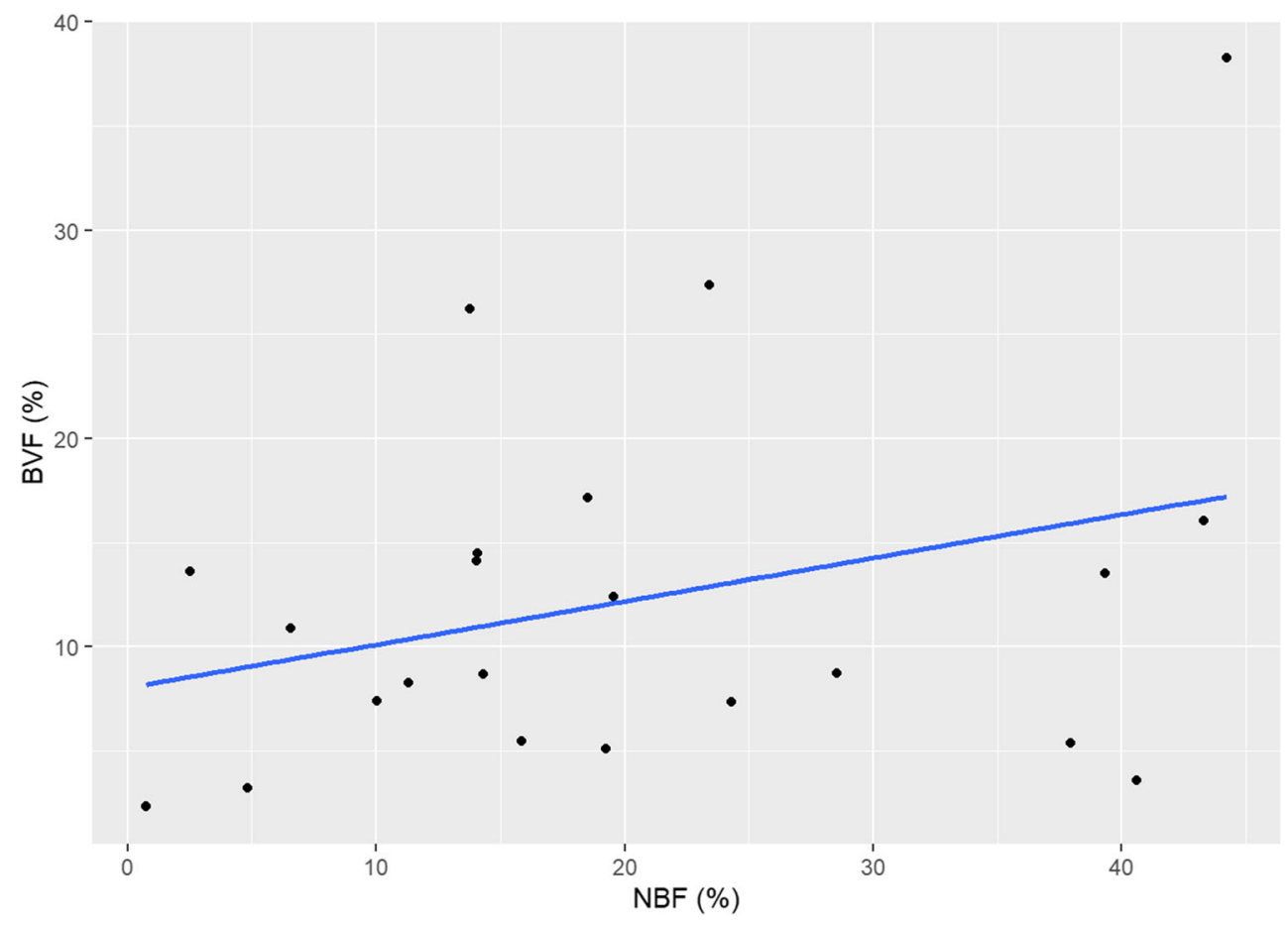





Fig. 11 Animal from group 2 weeks selected for visual comparison among the three assessment tools. a Coronal slice of DCE-MRI. Highlighted in green is the right defect, which was filled with an autogenous bone; in red, the left defect, filled with blood; in orange, an ROI of the brain, and in yellow, one of the masseter muscle, which was

DCE-MRI could also facilitate decision-making when pseudoarthrosis is suspected and the surgeon must decide whether to reintervene or not. This is a clinical-radiological diagnosis, which can be hard to establish, especially when the information from the two examinations is contradictory, e.g., a fracture line can no longer be identified on the X-ray, but the patient still feels pain or there still seems to be mobility between fragments. Should we be able to see signs of vessels sprouting from the proximal to the distal segment, bridging the gap, which would be another indicator of bone union.

As for repercussions on pre-clinical research, our study adds to a growing body of evidence that shows that MRI is effective in monitoring neovascularization during bone regeneration in different animal models $[15,17,19,20]$. Thus, future studies that aim at assessing the vascularization of bone grafts or bone tissue-engineered constructs can do so using MRI instead of histology. That could not only lead to a reduction in sample size and, with it, in costs, but also allow for an intra-individual longitudinal assessment.

\section{Conclusions}

DCE-MRI consistently allowed the identification of the craniotomies and the assessment of the blood perfusion of the osteoid within them. Nonetheless, these findings did not correlate to the formation of new bone on histomorphometry or $\mu$-CT, which speaks against the use of IAUC $_{140}$ as a surrogate for the efficacy of osteoplastic materials. Though we showed this method to be viable, for the validation of DCE-MRI for monitoring neovascularization during bone regeneration, and, possibly, of IAUC I $_{100}$ as a surrogate for the efficacy of osteoplastic materials, confirmatory studies are needed.
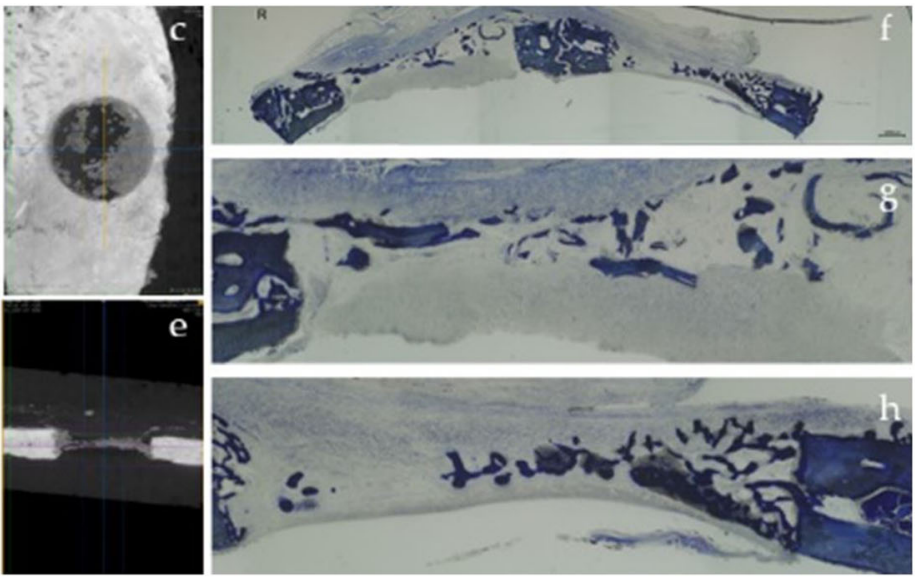

used as references to normalize the IAUC ${ }_{140}$ values for both defects; $\mathbf{b}$ a bird's view of the right and $\mathbf{c}$ left defects on $\mu$-CT. $\mathbf{d}$ A coronal slice of the right and e left defects on $\mu$-CT. $\mathbf{f} \mathrm{A} \times 2$ magnification of a slide containing both defects, stained with toluidine blue; $\mathbf{g}$ a close-up of the right and $\mathbf{h}$ left defects

Acknowledgements The authors would like to thank Dr. Jan Baumgart, Head of the Translational Animal Research Center (TARC) of the University Medical Center Mainz, for his help with the planning of the project, ensuring compliance to the ethical guidelines; Dr. Manfred Berres, a statistician from the Institute for Medical Biostatistics, Epidemiology and Informatics (IMBEI) of the University Medical Center Mainz, for his assistance in planning the project and conducting the statistical analysis; Dr. Vinay V. Kumar, Oral \& Maxillofacial Surgeon and Researcher of the Department of Plastic and Maxillofacial Surgery of the Uppsala University Hospital, Sweden, not only for his valuable insights in reviewing this paper but also, as a native speaker, for assuring a high standard of English; and Kerstin Bahr, veterinary staff from the Institute of Functional and Clinical Anatomy of the University Medical Center Mainz, for supervising the animals.

Funding Open Access funding enabled and organized by Projekt DEAL. The first author would like to declare the financial support received from Coordenação de Aperfeiçoamento de Pessoal de Nível Superior (CAPES) in the form of a PhD scholarship (Process: BEX 8956/14-6), as well as from the International Team for Implantology, in the form of a research grant (1077_2015). M. Terekhov and L. M. Schreiber obtained financial support from the German Ministry of Education and Research (BMBF), under grants 01EO1004 and 01EO1504.

\section{Declarations}

Ethical approval All applicable international, national, and/or institutional guidelines for the care and use of animals were followed.

Informed consent For this type of study, formal consent is not required.

Conflict of interest The first author would also like to disclose his ties with the AO Foundation, where he serves as an AO PEER Teacher, Adjunct Member of AO CMF ESA Community Development, Junior Researcher of the AO CMF R\&D Commission, and Member of the Support Group for Research Projects of AO Trauma Latin America, besides having been granted an Education Grant to attend the AO PEER Teacher Seminar in 2019 and having been a Research Fellow at AO CID in 2012. T. Emrich received speaker fees and travel support from Siemens 
Healthcare GmbH. M. Terekhov declares that he has no conflict of interest. H. Götz declares that he has no conflict of interest. M. Ackermann declares that he has no conflict of interest. L.M. Schreiber declares that she has no conflict of interest. W.E.G. Müller declares that he has no conflict of interest. J. Jung declares that he has no conflict of interest. J.P. Rojas declares that he has no conflict of interest. B. Al-Nawas declares that he has no conflict of interest.

Open Access This article is licensed under a Creative Commons Attribution 4.0 International License, which permits use, sharing, adaptation, distribution and reproduction in any medium or format, as long as you give appropriate credit to the original author(s) and the source, provide a link to the Creative Commons licence, and indicate if changes were made. The images or other third party material in this article are included in the article's Creative Commons licence, unless indicated otherwise in a credit line to the material. If material is not included in the article's Creative Commons licence and your intended use is not permitted by statutory regulation or exceeds the permitted use, you will need to obtain permission directly from the copyright holder. To view a copy of this licence, visit http://creativecommons.org/licenses/by/4.0/.

\section{References}

1. de Isla N, Huseltein C, Jessel N, Pinzano A, Decot V, Magdalou J, Bensoussan D, Stoltz JF (2010) Introduction to tissue engineering and application for cartilage engineering. Biomed Mater Eng 20(3): 127-133. https://doi.org/10.3233/BME-2010-0624

2. Kim JJ, Hou L, Huang NF (2016) Vascularization of threedimensional engineered tissues for regenerative medicine applications. Acta Biomater 41:17-26. https://doi.org/10.1016/j.actbio. 2016.06.001

3. Fetz AE, Radic MZ, Bowlin G (2020) Neutrophils in biomaterialguided tissue regeneration: matrix reprogramming for angiogenesis. Tissue Eng Part B Rev. https://doi.org/10.1089/ten.TEB.2020. 0028

4. Casanova MR, Oliveira C, Fernandes EM, Reis RL, Silva TH, Martins A, Neves NM (2020) Spatial immobilization of endogenous growth factors to control vascularization in bone tissue engineering. Biomater Sci 8:2577-2589. https://doi.org/10.1039/ d0bm00087f

5. Laschke MW, Menger MD (2017) Spheroids as vascularization units: from angiogenesis research to tissue engineering applications. Biotechnol Adv 35(6):782-791. https://doi.org/10.1016/j. biotechadv.2017.07.002

6. Son J, Kim J, Lee K, Hwang J, Choi Y, Seo Y, Jeon H, Kang HC, Woo HM, Kang BJ, Choi J (2019) DNA aptamer immobilized hydroxyapatite for enhancing angiogenesis and bone regeneration. Acta Biomater 99:469-478. https://doi.org/10.1016/j.actbio.2019. 08.047

7. Zheng ZW, Chen YH, Wu DY, Wang JB, Lv MM, Wang XS, Sun J, Zhang ZY (2018) Development of an accurate and proactive immunomodulatory strategy to improve bone substitute materialmediated osteogenesis and angiogenesis. Theranostics 8(19):54825500. https://doi.org/10.7150/thno.28315

8. Hillman H (2000) Limitations of clinical and biological histology. Med Hypotheses 54(4):553-564. https://doi.org/10.1054/mehy. 1999.0894
9. Udagawa A, Sato S, Hasuike A, Kishida M, Arai Y, Ito K (2013) Micro-CT observation of angiogenesis in bone regeneration. Clin Oral Implants Res 24(7):787-792. https://doi.org/10.1111/j.16000501.2012.02458.x

10. Young S, Kretlow JD, Nguyen C, Bashoura AG, Baggett LS, Jansen JA, Wong M, Mikos AG (2008) Microcomputed tomography characterization of neovascularization in bone tissue engineering applications. Tissue Eng B Rev 14(3):295-306. https://doi.org/ 10.1089/ten.teb.2008.0153

11. Klinck RJ, Campbell GM, Boyd SK (2008) Radiation effects on bone architecture in mice and rats resulting from in vivo microcomputed tomography scanning. Med Eng Phys 30(7):888-895. https://doi.org/10.1016/j.medengphy.2007.11.004

12. Choyke PL, Dwyer AJ, Knopp MV (2003) Functional tumor imaging with dynamic contrast-enhanced magnetic resonance imaging. J Magnet Reson Imag 17(5):509-520. https://doi.org/10.1002/ jmri.10304

13. Donath K (1988) Preparation of histologic sections by the cuttinggrinding technique for hard tissue and other material not suitable to be sectioned by routine methods. Equipment and methodical performance

14. Percie du Sert N, Hurst V, Ahluwalia A, Alam S, Avey MT, Baker M, Browne WJ, Clark A, Cuthill IC, Dirnagl U, Emerson M, Garner P, Holgate ST, Howells DW, Karp NA, Lazic SE, Lidster K, CJ MC, Macleod M, Pearl EJ, Petersen OH, Rawle F, Reynolds P, Rooney K, Sena ES, Silberberg SD, Steckler T, Wurbel H (2020) The ARRIVE guidelines 2.0: updated guidelines for reporting animal research. J Physiol. https://doi.org/10.1113/JP280389

15. Beaumont M, DuVal MG, Loai Y, Farhat WA, Sandor GK, Cheng HL (2010) Monitoring angiogenesis in soft-tissue engineered constructs for calvarium bone regeneration: an in vivo longitudinal DCE-MRI study. NMR Biomed 23(1):48-55. https://doi.org/10. $1002 / \mathrm{nbm} .1425$

16. Goldberg VM, Stevenson S (1987) Natural history of autografts and allografts. Clin Orthop Relat Res 225:7-16

17. Woloszyk A, Wolint P, Becker AS, Boss A, Fath W, Tian Y, Hoerstrup SP, Buschmann J, Emmert MY (2019) Novel multimodal MRI and micro-CT imaging approach to quantify angiogenesis and 3D vascular architecture of biomaterials. Sci Rep 9(1):19474. https://doi.org/10.1038/s41598-019-55411-4

18. Sauerbier S, Palmowski M, Vogeler M, Nagursky H, Al-Ahmad A, Fisch D, Hennig J, Schmelzeisen R, Gutwald R, Fasol U (2009) Onset and maintenance of angiogenesis in biomaterials: in vivo assessment by dynamic contrast-enhanced MRI. Tissue Eng C Meth 15(3):455-462. https://doi.org/10.1089/ten.tec.2008.0626

19. Ribot EJ, Tournier C, Aid-Launais R, Koonjoo N, Oliveira H, Trotier AJ, Rey S, Wecker D, Letourneur D, Amedee Vilamitjana J, Miraux S (2017) 3D anatomical and perfusion MRI for longitudinal evaluation of biomaterials for bone regeneration of femoral bone defect in rats. Sci Rep 7(1):6100. https://doi.org/10.1038/ s41598-017-06258-0

20. Song D, Shujaat S, Zhao R, Huang Y, Shaheen E, Van Dessel J, Orhan K, Vande Velde G, Coropciuc R, Pauwels R, Politis C, Jacobs R (2020) In vivo quantification of mandibular bone remodeling and vascular changes in a Wistar rat model: a novel HR-MRI and micro-CT fusion technique. Imag Sci Dent 50(3):199-208. https://doi.org/10.5624/isd.2020.50.3.199

Publisher's note Springer Nature remains neutral with regard to jurisdictional claims in published maps and institutional affiliations. 